\title{
CONE CHARACTERIZATION OF REFLEXIVE BANACH LATTICES
}

\author{
by IOANNIS A. POLYRAKIS
}

(Received 18 June, 1993)

\begin{abstract}
We prove that a Banach lattice $X$ is reflexive if and only if $X_{+}$does not contain a closed normal cone with an unbounded closed dentable base.
\end{abstract}

Suppose that $X$ is a Banach space and $P$ a cone of $X$ (i.e. $P \subseteq X, \lambda P+\mu P=P$ for each $\lambda, \mu \in \mathbb{R}_{+}$and $P \cap(-P)=\{0\}$ ). The cone $P$ is normal (or self-allied) if there exists $a \in \mathbb{R}_{+}$such that for each $x, y \in P, x \leq y$ implies $\|x\| \leq a\|y\|$. A convex subset $B$ of $P$ is a base for $P$ if for each $x \in P, x \neq 0$, there exists a unique number $f(x) \in \mathbb{R}_{+}$such that $(f(x))^{-1} x \in B$. For each $D \subseteq X$, denote by co $D$ the closed convex hull of $D$. A subset $K$ of $X$ is dentable if for each $\varepsilon \in \mathbb{R}_{+}$there exists $x_{\varepsilon} \in K$ such that $x_{\varepsilon} \notin \overline{\operatorname{co}}\left\{x \in K \mid\left\|x-x_{\varepsilon}\right\| \geq\right.$ $\varepsilon\}$.

We say that the cone $P$ of $X$ is isomorphic (or according to [6] and [7], locally isomorphic) to a cone $Q$ of a Banach space $Y$ if there exists an one-to-one, additive, positive homogeneous map $T$ of $P$ onto $Q$ and $T, T^{-1}$ are continuous in the induced topologies. Denote by $c_{0}$ the space of convergent to zero real sequences with the supremum norm and by $l_{1}$ the space of absolutely summing real sequences $\xi=(\xi(i))$ with the norm $\|\xi\|=\sum_{i=1}^{\infty}|\xi(i)|$. The cones

$$
\begin{aligned}
& c_{0}^{+}=\left\{x=(x(i)) \in c_{0} \mid x(i) \in \mathbb{R}_{+} \text {for each } i\right\}, \\
& l_{1}^{+}=\left\{x=(x(i)) \in l_{1} \mid x(i) \in \mathbb{B}_{+} \text {for each } i\right\},
\end{aligned}
$$

are the positive cones of $c_{0}, l_{1}$ respectively. If $l_{1}^{+}$(respectively $c_{0}^{+}$) is isomorphic to a closed cone $D \subseteq P$, then we say that $l_{1}^{+}$(respectively $c_{0}^{+}$) is embeddable in $P$. Cones isomorphic to $l_{1}^{+}$are studied in [6]. For notation and terminology on convex sets we refer to [2].

TheOREM 1 ([5, Theorem 1]). Let $X$ be a reflexive Banach space. Then $X$ does not contain a closed normal cone with an unbounded closed dentable base.

Let $X$ be a Banach lattice. By G. Lozanovskii's Theorem, see [4] or [1, p. 240], $X$ is reflexive if and only if neither $c_{0}$ or $l_{1}$ is lattice embeddable in $X$.

THEOREM 2. Let $X$ be a Banach lattice. Then the following statements are equivalent:

(i) $X$ is reflexive,

(ii) $l_{1}^{+}$is not embeddable in $X_{+}$,

(iii) $X_{+}$does not contain a closed normal cone $P$ with an unbounded closed dentable base.

Proof. By Theorem 1, (i) $\Rightarrow$ (iii). Let the statement (iii) be true. Suppose that the statement (ii) does not hold. Then there exists a closed cone $P$ of $X$ isomorphic to $l_{1}^{+}$and 
let $T: l_{1}^{+} \rightarrow P$ be an isomorphism. By the continuity of $T$ and $T^{-1}$ at zero, there exist $a$, $b \in \mathbb{R}_{+}$such that

$$
a\|x\| \leq\|T(x)\| \leq b\|x\|, \quad \text { for each } x \in l_{1}^{+} .
$$

Let $f=(\xi(k))$ with $\xi(k)=k^{-1}$ for each $k \in \mathbb{N}$. The set $B=\left\{x \in l_{1}^{+} \mid f(x)=1\right\}$ is a closed base for $l_{1}^{+}$and $T(B)$ a closed base for $P$. The cone $P$ is normal because it is contained in $X$. The base $B$ is unbounded because $k e_{k} \in B$ for each $k \in \mathbb{N}$; therefore $T(B)$ is also unbounded. The functional $g=(q(i))$ with $q(1)=1$ and $q(k)=-1$ for each $k \neq 1$, strongly exposes the point $e_{1}=(1,0,0, \ldots)$ in $B$. This holds because if $x \in B$ with $x \neq e_{1}$, then

$$
g(x)=x(1)-\sum_{k=2}^{\infty} x(k)<x(1)<f(x)=g\left(e_{1}\right) .
$$

Also, if $x_{n} \in B$ with $g\left(x_{n}\right)=x_{n}(1)-\sum_{k=2}^{\infty} x_{n}(k) \rightarrow 1$, then $x_{n}(1) \rightarrow 1$ and $\sum_{k=2}^{\infty} x_{n}(k) \rightarrow 0$; therefore $\left\|e_{1}-x_{n}\right\| \rightarrow 0$. Let $z_{1}=T\left(e_{1}\right)$ and $h(y)=g\left(T^{-1}(y)\right)$, for each $y \in P$. Then $h(y)<h\left(z_{1}\right)$ for each $y \in T(B)$ with $y \neq z_{1}$. For each sequence $y_{n}=T\left(x_{n}\right)$ of $T(B)$ with $h\left(y_{n}\right) \rightarrow h\left(z_{1}\right)$ we have that $g\left(x_{n}\right) \rightarrow g\left(e_{1}\right)$; therefore $x_{n} \rightarrow e_{1}$ and so $y_{n} \rightarrow z_{1}$. Thus for each $\varepsilon \in \mathbb{R}_{+}$there exists $\rho=\rho(\varepsilon) \in \mathbb{R}_{+}$such that $h(y)<h\left(z_{1}\right)-\rho$, for each $y \in T(B)$ with $\left\|y-z_{1}\right\| \geq \varepsilon$. Since $h$ is additive, positive homogeneous and continuous we have

$$
h(y) \leq h\left(z_{1}\right)-\rho, \quad \text { for each } y \in \overline{\operatorname{co}}\left\{z \in T(B) \mid\left\|z-z_{1}\right\| \geq \varepsilon\right\},
$$

therefore $T(B)$ is dentable. This is a contradiction; therefore (iii) $\Rightarrow$ (ii).

Suppose now that the statement (ii) holds. Since $X_{+}$does not contain $l_{1}^{+}$we have that $l_{1}$ is not lattice embeddable in $X$. Let $b_{n}=\sum_{i=1}^{n} e_{i}$, where $\left(e_{n}\right)$ is the usual (Schauder) basis of $c_{0}$. Then $\left(b_{n}\right)$ is a basis of $c_{0}$ because for each $x=(x(i)) \in c_{0}$ we have

$$
\sum_{i=1}^{n}(x(i)-x(i+1)) b_{i}=\sum_{i=1}^{n} x(i) e_{i}-x(n+1) b_{n} \text { and } \lim _{n \rightarrow \infty} x(n+1) b_{n}=0 .
$$

The basis $\left(b_{n}\right)$ is of type $l_{+}$(i.e. $\left(b_{n}\right)$ is bounded and there exists $k \in \mathbb{R}_{+}, k \neq 0$ such that $\left\|\sum_{i=1}^{n} a_{i} b_{i}\right\| \geq k \sum_{i=1}^{n} a_{i}$, for each finite sequence $a_{1}, a_{2}, \ldots, a_{n}$, of positive real numbers); therefore by [7, Theorem II.10.2, p. 323], the positive cone

$$
C=\left\{\sum_{i=1}^{\infty} \lambda_{i} b_{i} \in c_{0} \mid \lambda_{i} \in \mathbb{R}_{+} \text {for each } i\right\} \subseteq c_{0}^{+}
$$

of the basis $\left(b_{n}\right)$, is isomorphic to $l_{1}^{+}$. ( $C$ is the set of decreasing real sequences convergent to zero.) This shows that $c_{0}$ is not lattice embeddable in $X$; therefore $X$ is reflexive.

REMARK 1 . In the proof of the previous theorem we have also show that $l_{1}^{+}$is isomorphic to the cone $C \subseteq c_{0}^{+}$, of decreasing real sequences convergent to zero; therefore $l_{1}^{+}$is embeddable in $c_{0}^{+}$.

It is known [1, Theorem 14.12, p. 226] that a Banach lattice $X$ is a KB-space (i.e. $X$ has the property: every increasing, norm bounded, sequence of $X_{+}$is norm convergent) if 
and only if $c_{0}$ is not lattice embeddable in $X$. Also $c_{0}^{+}$is not embeddable in the positive cone $X_{+}$of a KB-space. This holds because if we suppose that a closed cone $P \subseteq X_{+}$is isomorphic to $c_{0}^{+}$, and $T: c_{0}^{+} \rightarrow P$ is an isomorphism then we have: the sequence $s_{n}=T\left(b_{n}\right)$, where $b_{n}=\sum_{i=1}^{n} e_{i} \in c_{0}^{+}$, is norm bounded because $\left\|T\left(b_{n}\right)\right\| \leq A\left\|b_{n}\right\|=A$, for each $n$. $\left(s_{n}\right)$ is also increasing; therefore $\left(s_{n}\right)$ is norm convergent to a point $s$ of $P$. If $T(e)=s$, then $b_{n} \rightarrow e$, which is a contradiction; therefore $c_{0}^{+}$is not embeddable in $X_{+}$. Now, using Theorem 2 and the above remarks we obtain the following characterization of Banach lattices $X$ in terms of the embeddability of the cones $l_{1}^{+}$and $c_{0}^{+}$in $X_{+}$.

THEOREM 3. A Banach lattice $X$ is a non-reflexive $K B$-space if and only if $l_{1}^{+}$is embeddable in $X_{+}$and $c_{0}^{+}$is not embeddable in $X_{+}$.

\section{REFERENCES}

1. C. D. Aliprantis and O. Burkinshaw, Positive operators (Academic Press, 1985).

2. J. Diestel and J. J. Uhl, Vector measures (Math. Surveys, Vol. 15. Amer. Math. Soc., 1977).

3. G. J. O. Jameson, Ordered linear spaces, Lecture Notes in Mathematics No 141 (Springer-Verlag, 1970).

4. G. Ya. Lozanovskĩ, Banach structures and bases, Functional Analysis and its Applictions 1 (1967), 249.

5. I. A. Polyrakis, Extreme points of unbounded, closed and convex sets in Banach spaces, Math. Proc. Cambridge Phil. Soc. 95 (1984), 319-323.

6. I. A. Polyrakis, Cones locally isomorphic to the positive cone of $l_{1}(\Gamma)$, Linear Algebra and Appl. 84 (1986), 323-334.

7. I. Singer, Bases in Banach spaces I (Springer-Verlag, 1974).

Department of Mathematics

National Technical University

Zografou Campus

15780 ATHENS

GREECE

e-mail: ypoly@hisyros.ntua.gr 